\title{
Structural studies on lamin
}

\author{
Similarities and differences between lamin and intermediate-filament proteins
}

\author{
David A. D. PARRY,* James F. CONWAY* and Peter M. STEINERT $\dagger$ \\ *Department of Physics and Biophysics, Massey University, Palmerston North, New Zealand, and †Dermatology Branch, \\ National Cancer Institute, Building 10, Room 12N238, National Institutes of Health, Bethesda, MD 20892, U.S.A.
}

\begin{abstract}
Analysis of the amino acid sequences of lamins $A$ and $C$ has revealed that each chain has an almost continuous heptad-containing coiled-coil domain containing structural regularities in the linear disposition of the acidic and the basic residues. The data suggest that the lamin molecules are two-stranded ropes, that the two chains are parallel to one another and in axial register, and that the molecules aggregate in vivo through periodic ionic interactions. These results indicate that significant changes in stability of the nuclear envelope may be achieved between interphase and mitosis through changes in the degree of phosphorylation of the lamin proteins.
\end{abstract}

\section{INTRODUCTION}

The structural integrity of the nuclear envelope is at least in part attributable to the nuclear lamina complex, which, in mammals, is composed of three structural protein chains, lamins A, B and C (Dwyer \& Blobel, 1976; Gerace \& Blobel, 1980; Gerace, 1985). Lamins A and $\mathrm{C}$ differ in molecular mass by about $10 \mathrm{kDa}$ but are identical except for their $C$-terminal sequences (McKeon et al., 1986). Lamin B, however, is significantly different from both on the basis of peptide mapping and immunological properties (Gerace \& Blobel, 1980; Shelton et al., 1980). These three proteins form a complex fibrous network on the inner surface of the nucleus that may be continuous with the nuclear pores, and is thought to anchor interphase chromosomes (Dwyer \& Blobel, 1976; Gerace, 1985). During mitosis, this network undergoes major structural changes: concomitant with a wave of phosphorylation of the lamins, the network disintegrates soon after chromosome condensation at prophase, and is reassembled at telophase simultaneously with dephosphorylation of the lamins (Miake-Lye \& Kirschner, 1985; Burke \& Gerace, 1986).

The sequences of lamins $\mathrm{A}$ and $\mathrm{C}$ have been shown to bear a remarkable resemblance to the primary structures of the five classes of intermediate-filament (IF) proteins (keratin, desmin, vimentin, glial fibrillary acidic protein and neurofilament protein), and on these grounds it was postulated that IF and lamin molecules would have structural and aggregation properties in common (Gerace, 1985; McKeon et al., 1986). Since IF structure has been extensively studied and a relationship between IF and lamin chains has been established, there are some grounds for optimism that it may now be possible to understand how the nuclear envelope is able to maintain high stability in interphase while still being capable of total disassembly and subsequent reassembly during mitosis.

\section{RESULTS AND DISCUSSION}

In order to assess the degree of similarity and difference between the IF and lamin chains, it is useful to restate the main features of IF chain structures (Parry \& Fraser, 1985; Steinert \& Parry, 1985). Firstly, IF protein chains may be divided into non- $\alpha$-helical, basic $N$-terminal and $C$-terminal domains of variable chemical character and size enclosing a homologous and generally acidic $\alpha$-helix-rich rod domain. This latter domain can be further subdivided into four heptad-containing segments (1A, 1B, 2A and 2B) and three non-heptad-containing linking segments (L1, L12 and L2) (Fig. 1a). Two parallel chains, each with a coiled-coil conformation, aggregate in axial register to form the IF protein molecule. Secondly, on the basis of the extent of homology within the rod domain, IF protein chains may be classified as type I, II, III or IV: keratin filaments contain both 'acidic' type I chains ( $\mathrm{pI}<5.5)$ and 'neutral-basic' type II chains ( $\mathrm{pI}>6$ ); desmin, vimentin and glial fibrillary acidic protein filaments contain only type III chains; neurofilaments contain only type IV chains. Thirdly, all IF protein chains contain a highly conserved sequence in segment $1 \mathrm{~A}$ and another at the $C$-terminal end of segment $2 B$. Also, there is a conserved break in the phasing of the heptad substructure towards the middle of segment 2B. Fourthly, segment $1 B$ and segment $2(=2 \mathrm{~A}+\mathrm{L} 2+2 \mathrm{~B})$ each contain a highly significant period of about 9.55 and 9.84 residues respectively in the linear disposition of both the acidic residues and the basic residues. Such regularities provide the means by which periodic salt bridges may be formed between positively and negatively charged residue groupings; these stabilize molecular aggregates and specify the appropriate axial relationships between them in the IF (Parry \& Fraser, 1985).

Let us now compare the sequence and predicted structure of lamins $A$ and $C$ with that of the IF proteins. The methods employed are discussed in detail by Parry

Abbreviation used: IF, intermediate filament.

Vol. 238 
$1 \mathrm{~A}$

$1 \mathrm{~B}$

2A

2B

(a)

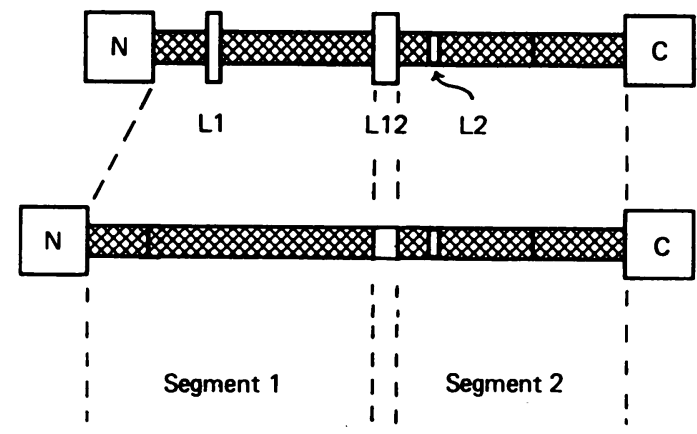

Fig. 1. Schematic representations of the structures of IF protein and lamin

(a) Schematic representation of the structure of an IF protein chain (Parry \& Fraser, 1985, and references cited therein). $\mathbf{N}$ and $\mathbf{C}$ represent the variable non- $\alpha$-helical and generally basic domains at each end of the chain. The intervening rod domain, rich in $\alpha$-helical structure and with a correspondingly high proportion of heptad substructure characteristic of a coiled-coil conformation (shown cross-hatched), can be divided into segment 1 $(1 \mathrm{~A}+\mathrm{L} 1+1 \mathrm{~B}$; length $22 \mathrm{~nm})$ and segment 2 $(2 \mathrm{~A}+\mathrm{L} 2+2 \mathrm{~B}$; length $22 \mathrm{~nm})$ separated by a non-helical link L12. The former consists of an $\alpha$-helical segment 1A with heptad repeat, a non- $\alpha$-helical link L1 of variable size and an $\alpha$-helical segment 1B with heptad repeat. Segment 2 consists of $\alpha$-helical segment $2 A$ with heptad repeat, an $\alpha$-helical link $L 2$ of fixed length that lacks a heptad repeat and $\alpha$-helical segment $2 B$ with a heptad repeat containing a discontinuity (as marked) approximately at its mid-point. Segments $1 \mathrm{~B}$ and 2 each contain a regular linear disposition of acidic and basic residues; the periods in segments $1 B$ and 2 are 9.55 and 9.84 residues respectively. (b) Schematic representation of the structures of lamins A and C. Segments 1A, 2A, L2 and 2B are identical in length with those in IF chains and are homologous to them. Further, the sizes of $\mathrm{N}, \mathrm{C}, \mathrm{Ll}$ and $\mathrm{L} 12$ lie in or very close to the ranges found in IF chains. Segment $1 \mathrm{~B}$, however, is six heptads (42 residues) longer in lamin than in IF chains. Furthermore, a heptad substructure exists from the $N$-terminus of segment $1 \mathrm{~A}$ lthrough the $\alpha$-helical segment $\mathrm{Ll}$ to the $C$-terminus of segment $1 \mathrm{~B}$, thus generating a coiled-coil segment (segment 1) of length $28 \mathrm{~nm}$. A heptad discontinuity, however, is found in segment $\mathrm{Ll}$ (as marked). The structure of segment $\mathrm{L} 12$ is predicted to be $\alpha$-helical, though it lacks the characteristic heptad repeat existing in all remaining portions of the rod domain other than L2. Segment 1 (length $28 \mathrm{~nm}$ ) and segment 2 (length $22 \mathrm{~nm}$ ) each contain regularities in their linear distributions of the acidic and basic residues (Table 1), and these regularities are maintained in phase across the link L12, thus generating a continuous rod of length $53 \mathrm{~nm}$.

\& Fraser (1985) and include the secondary-structure prediction methods of Chou \& Fasman (1974) and Garnier et al. (1978). The structural similarities, some of which have already been reported by McKeon et al. (1986), are remarkable, and may be summarized as follows: (i) both chain species have largely non- $\alpha$-helical, basic $N$-terminal and $C$-terminal domains enclosing an $\alpha$-helix-rich rod domain; (ii) the rod domains of each can be divided into homologous segments $(1 \mathrm{~A}, 2 \mathrm{~A}, \mathrm{~L} 2$ and
2B) and those that are analogous (L1, 1B and L12); (iii) each rod domain contains a highly conserved sequence in segment $1 \mathrm{~A}$ and at the $C$-terminal end of segment $2 \mathrm{~B}$; (iv) interchain ionic interactions lead to an in-register arrangement of chains in both lamin and IF to form two-chain coiled-coil molecules. The differences between lamins $\mathrm{A}$ and $\mathrm{C}$ and the IF proteins are equally marked, and these may be summarized as folows: (i) segment $1 \mathrm{~B}$ in the lamin chains is six heptads (i.e. 42 and not 43 residues as reported by McKeon et al., 1986) longer than in the IF proteins (Fig. 1b); the 42-residue insert is part of a 70-residue piece in lamins $\mathrm{A}$ and $\mathrm{C}$ that replaces residues 43-70 inclusive in segment $1 B$ of IF chains (note that it occurs at the position of a conserved intron; Steinert et al., 1985; see also Weber, 1986); also, segment $1 B$ in lamin chains is highly charged but neutral (30 acidic and 30 basic residues) and not strongly acidic as in IF chains (23 acidic and 16 basic residues, on average); (ii) segment 2 is more highly charged in lamin chains ( 32 acidic and 26 basic residues) than in IF chains (29 acidic and 22 basic residues, on average); (iii) segment L12 is predicted to be $\alpha$-helical in lamin chains but non- $\alpha$-helical in IF chains; neither chain species has a heptad repeat; (iv) segment $L 1$ in lamin has a heptad substructure and is also predicted to be $\alpha$-helical; thus a continuous heptad substructure extends from the $N$-terminal end of segment $1 \mathrm{~A}$ to the $C$-terminal end of segment 1B, though a single 'stutter' or break in heptad phasing does occur in segment Ll (cf. segment 2B); (v) the significant periods in the linear distribution of the acidic and the basic residues in segments 1 and 2 in lamin chains differ slightly from those in segments $1 B$ and 2 in IF chains (Table 1); (vi) the number of stabilizing interchain ionic interactions for a parallel in-register arrangement of chains is much higher for lamin molecules than it is for the IF protein molecules [cf. 36 interactions with 8-18 in IF protein molecules (Table 2)]; (vii) on the basis of homology of the rod domain segments lamin chains do not fall naturally into the type I, II, III or IV classification; they may, however, be termed type $\mathrm{V}$ chains, since they do exhibit considerable homology to IF chains in general (Parry \& Fraser, 1985; Gerace, 1985; Steinert \& Parry, 1985; Steinert et al., 1985); (viii) unlike types I-IV IF chains lamins do not form long filamentous structures (i.e. IF) in vitro under any known conditions; instead they form tactoids or paracrystalline arrays (Zackroff et al., 1984; Goldman et al., 1986) of alternating dark- and light-staining bands of period approx. $35 \mathrm{~nm}$; these have been interpreted as arrays of $\alpha$-helical rod domains separated by non- $\alpha$ helical 'globular' domains.

Comparison of the data from IF proteins and lamin chains allows a number of predictions to be made about the structure of the latter.

(a) The lamin molecules will be two-stranded coiledcoil ropes and will contain parallel chains in axial register; the overall length of the rod domain of the lamin molecule (356 residues) will be about $356 \times 0.1485 \mathrm{~nm}=53 \mathrm{~nm}$, a value significantly greater than for an IF molecule (about $47 \mathrm{~nm}$ ).

(b) As judged from the greater number of potential interchain ionic interactions individual lamin molecules are likely to be more stable overall than the IF molecules; this suggests the likelihood of a looser and more dynamic arrangement of molecular aggregation than in IF, possibly in the form of a mesh-like network. 
Table 1. Selection of the most significant structural periodicities in the linear disposition of the acidic and the basic residues in segments 1 and 2 of lamins $A$ and $C$

Acidic residues are aspartic acid and glutamic acid; basic residues are arginine and lysine. The probability of a particular period occurring by chance is given by the expression $P=\exp$ (-Intensity). The values of $P_{1}, P_{2}, P_{3}$ and $P_{4}$ are $9.94,9.29,10.02$ and 10.12 residues respectively. In IF chains the mean periodicity in the acidic and in the basic residues is 9.56 residues in segment $1 B$ and 9.84 residues in segment 2. Fourier intensities less than 5.0, where these may be of significance, are given in parentheses.

\begin{tabular}{|c|c|c|c|c|c|c|c|}
\hline \multicolumn{4}{|c|}{ Segment 1} & \multicolumn{4}{|c|}{ Segment 2} \\
\hline \multicolumn{2}{|c|}{ Acidic } & \multicolumn{2}{|c|}{ Basic } & \multicolumn{2}{|c|}{ Acidic } & \multicolumn{2}{|c|}{ Basic } \\
\hline Period & Intensity & Period & Intensity & Period & Intensity & Period & Intensity \\
\hline $\begin{array}{l}9.94\left(\mathrm{P}_{1}\right) \\
3.10 \\
2.34\end{array}$ & $\begin{array}{l}6.22 \\
5.25 \\
7.06\end{array}$ & $\begin{array}{l}(9.94) \\
9.29\left(\mathrm{P}_{2}\right) \\
3.02 \\
(2.32)\left(\mathrm{P}_{2} / 4\right) \\
(2.28)\end{array}$ & $\begin{array}{c}(3.49) \\
6.73 \\
8.58 \\
(3.00) \\
(4.75)\end{array}$ & $\begin{array}{l}(9.97)\left(\mathbf{P}_{3}\right) \\
(8.57) \\
(3.72) \\
(3.57) \\
(3.35)\left(P_{3} / 3\right) \\
(2.5)\left(P_{3} / 4\right) \\
(2.43) \\
2.12\end{array}$ & $\begin{array}{l}(2.33) \\
(4.03) \\
(3.52) \\
(4.09) \\
(3.06) \\
(3.88) \\
(3.74) \\
6.16\end{array}$ & $\begin{array}{l}(9.80) \\
5.06\left(\mathrm{P}_{4} / 2\right) \\
2.53\left(\mathrm{P}_{4} / 4\right) \\
(2.05)\left(\mathrm{P}_{4} / 5\right)\end{array}$ & $\begin{array}{c}(2.91) \\
7.55 \\
6.22 \\
(4.09)\end{array}$ \\
\hline
\end{tabular}

Table 2. Interchain ionic interactions as a function of relative axial stagger between identical similarly directed lamin $\mathbf{A}$ or $\mathbf{C}$ and also IF protein chains

H50K (K14; Marchuk et al., 1984) and H56K (K6a; Hanukoglu \& Fuchs, 1983) are respectively type I and type II chains from human epidermal keratin; CGD (Geisler \& Weber, 1982), HELV (Quax-Jeuken et al., 1983) and MGFAP (Lewis et al., 1984) are type III chains from chicken gizzard desmin, hamster eye lens vimentin and mouse glial fibrillary acidic protein respectively; PNF-L (Geisler et al., 1985b) is the type IV chain from the light component of pig neurofilament; lamin A or C is a type $\mathrm{V}$ chain from the nuclear lamina.

\begin{tabular}{|c|c|c|c|c|c|c|c|}
\hline $\begin{array}{l}\text { Stagger } \\
\text { (heptads) }\end{array}$ & $\begin{array}{l}\text { Lamin } A \text { or } C- \\
\text { lamin } A \text { or } C\end{array}$ & $\begin{array}{l}\text { H50K- } \\
\text { H50K }\end{array}$ & $\begin{array}{l}\text { H56K- } \\
\text { H56K }\end{array}$ & $\begin{array}{l}\text { CGD- } \\
\text { CGD }\end{array}$ & $\begin{array}{l}\text { HELV- } \\
\text { HELV }\end{array}$ & $\begin{array}{l}\text { MGFAP- } \\
\text { MGF AP }\end{array}$ & $\begin{array}{l}\text { PNF-L- } \\
\text { PNF-L }\end{array}$ \\
\hline $\begin{array}{r}0 \\
\pm 1 \\
\pm 2\end{array}$ & $\begin{array}{r}36 \\
-4 \\
8\end{array}$ & $\begin{array}{l}8 \\
5 \\
4\end{array}$ & $\begin{array}{r}12 \\
6 \\
3\end{array}$ & $\begin{array}{r}14 \\
2 \\
-4\end{array}$ & $\begin{array}{r}16 \\
5 \\
0\end{array}$ & $\begin{array}{r}18 \\
1 \\
-8\end{array}$ & $\begin{array}{r}12 \\
-1 \\
1\end{array}$ \\
\hline
\end{tabular}

(c) Molecular aggregation via ionic interactions between an antiparallel segment 1 -segment 1 combination and an antiparallel segment 2 -segment 2 combination is likely to be as important between lamin molecules as between IF molecules (Fraser et al., 1985; Fig. 2). Significant interactions between an antiparallel segment 1-segment 2 combination also appear to be likely between lamin molecules (Fig. 2), since the periods in the acidic residues and the basic residues are similar in both segment 1 and segment 2 . This would favour (i) an antiparallel arrangement of two molecules with segments 1 and 2 overlapped by an extent consistent with the calculated ionic interactions [Fig. 2; see also the proposals for IF in general (Fraser et al., 1985; Steinert \& Steven, 1985; Steinert et al., 1985) and desmin IF in particular (Geisler et al., 1985a)], and (ii) a parallel or antiparallel alignment of molecules that are approximately half-staggered with respect to one another (cf. IF). The interactions in (i) and (ii), which would be of the type 1U-2D (twice) and 1U-2U, 1U-1D or 2U-2D (respectively where $U$ and $D$ refer to 'up'- and 'down'-pointing segments), would be expected to correspond to one of the favoured sets of calculated ionic interactions (Fig. 2). Note also that the phasing of the acidic and the basic residues is maintained across the link L12, thus further suggesting that L12 is $\alpha$-helical. In the rod domain as a whole the periodicities in the acidic residues $(9.90$ residues, $I=6.29)$ and the basic residues (9.94 residues, $I=4.22$ ) differ in phase by about $150^{\circ}$.

(d) Higher order of molecular organization could occur via an antiparallel overlap of segment $1 \mathrm{~A}$ and an antiparallel overlap of the last four heptads in segment 2B, since these regions are the only ones that are preserved in toto in both lamin and IF proteins. In addition, elongation of the molecular aggregates into a network could occur by interactions between the terminal domains of the molecules.

(e) A model for lamin structure may be proposed in which neighbouring pairs of lamin molecules interact by means of their end domains and possibly portions of the rod domains to form an open mesh-like arrangement. Thus phosphorylation of the lamin chains during mitosis in either the rod domain or more probably the $N$ - and $C$-terminal domains may destabilize these interactions and dissociate the mesh. In this way interactions between lamin molecules and associated proteins, organelles etc. 

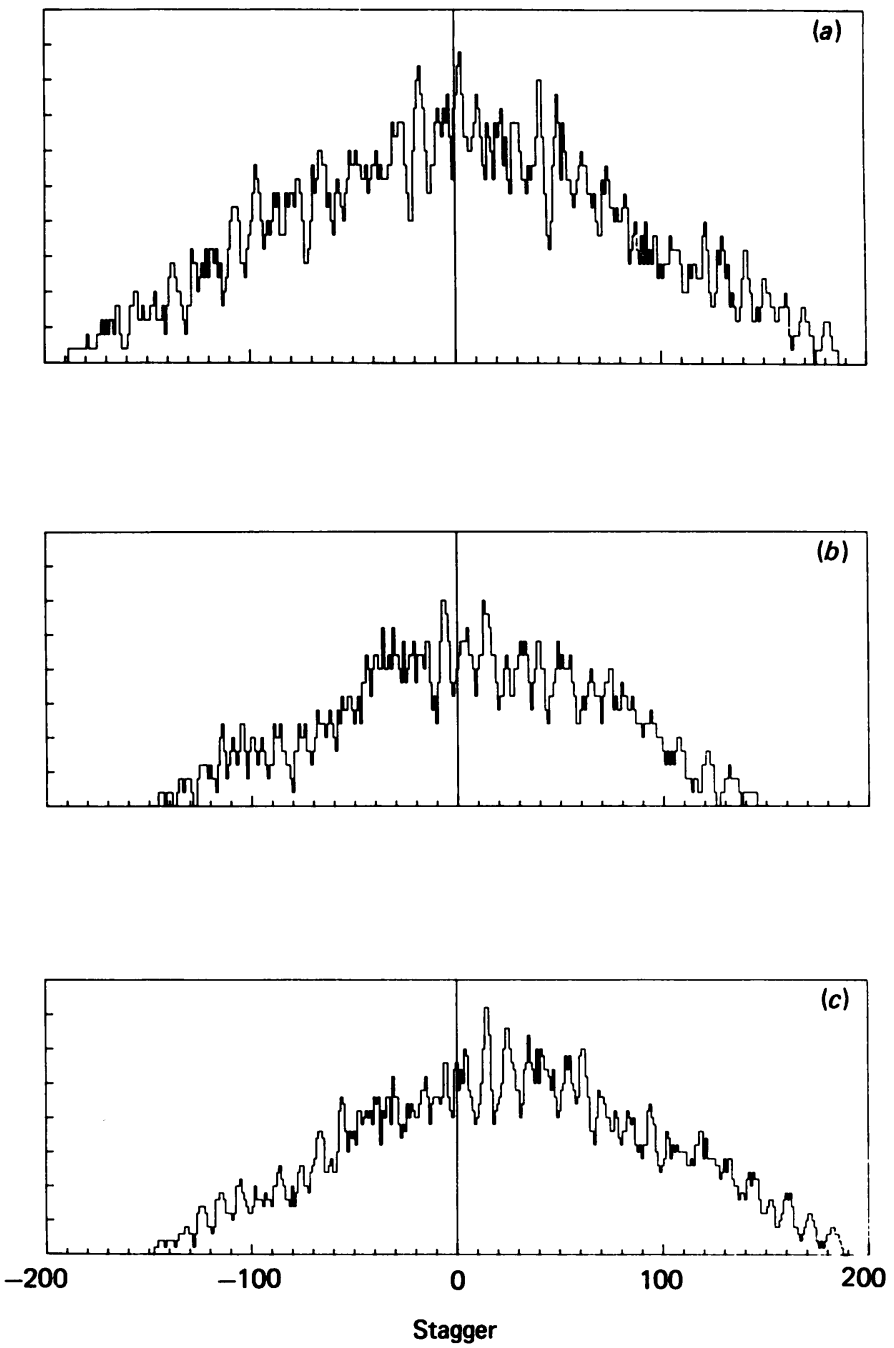

Fig. 2. Calculation of the possible number of ionic interactions as a function of stagger between (a) an antiparallel segment 1-segment 1 combination (1U-1D), (b) an antiparallel segment 2-segment 2 combination (2U-2D) and (c) an antiparallel segment 1 -segment 2 combination (1U-2D)

An ionic interaction is scored when a negatively charged residue (aspartic acid, glutamic acid) in one molecule lies within the axial range \pm 1 residue of a positively charged residue (arginine, lysine) in a second molecule (Fraser $e t$ al., 1985). Significant maxima can be seen, some of which will be important in specifying the modes of molecular aggregation in vivo. The lamin molecule is assumed here to have a homodimeric structure.

Received 13 May 1986/10 June 1986; accepted 17 June 1986 would be significantly modified. Dephosphorylation of the lamins would be expected to permit spontaneous reassembly of the mesh.

Further detailed structural analysis will be required to corroborate these predictions, but the notable similarities and differences between the lamins and the IF proteins make such studies potentially of great signifiance in both fields of study.

\section{REFERENCES}

Burke, B. \& Gerace, L. (1986) Cell (Cambridge, Mass.) 44, 639-652

Chou, P. Y. \& Fasman, G. D. (1974) Biochemistry 13, 211-245

Dwyer, N. \& Blobel, G. (1976) J. Cell Biol. 70, 581-591

Fraser, R. D. B., MacRae, T. P., Suzuki, E. \& Parry, D. A. D. (1985) Int. J. Biol. Macromol. 7, 258-274

Garnier, J., Osguthorpe, D. J. \& Robson, B. (1978) J. Mol. Biol. 120, 97-120

Geisler, N. \& Weber, K. (1982) EMBO J. 1, 1649-1656

Geisler, N., Kaufmann, E. \& Weber, K. (1985a) J. Mol. Biol. 182, $173-177$

Geisler, N., Plessmann, U. \& Weber, K. (1985b) FEBS Lett. 182, 475-478

Gerace, L. (1985) Nature (London) 318, 508-509

Gerace, L. \& Blobel, G. (1980) Cell (Cambridge, Mass.) 19, 277-287

Goldman, A. E., Maul, G., Steinert, P. M., Yang, H.-Y. \& Goldman, R. D. (1986) Proc. Natl. Acad. Sci. U.S.A. 83, in the press

Hanukoglu, I. \& Fuchs, E. (1983) Cell (Cambridge, Mass.) 33, 915-924

Lewis, S. A., Balcarek, J. M., Krek, V., Shelanski, M. \& Cowan, N. J. (1984) Proc. Natl. Acad. Sci. U.S.A. 81, 2743-2746

Marchuk, D., McCrohon, S. \& Fuchs, E. (1984) Cell (Cambridge, Mass.) 39, 491-498

McKeon, F. D., Kirschner, M. W. \& Caput, D. (1986) Nature (London) 319, 463-468

Miake-Lye, R. \& Kirschner, M. W. (1985) Cell (Cambridge, Mass.) 41, 165-175

Parry, D. A. D. \& Fraser, R. D. B. (1985) Int. J. Biol. Macromol. 7, 203-213

Quax-Jeuken, Y. E. F. M., Quax, W. J. \& Bloemendal, H. (1983) Proc. Natl. Acad. Sci. U.S.A. 80, 3548-3552

Shelton, K., Higgins, D., Cochran, D., Ruffolo, D. J. \& Egle, P. (1980) J. Biol. Chem. 255, 10976-10983

Steinert, P. M. \& Parry, D. A. D. (1985) Annu. Rev. Cell Biol. 1, 41-65

Steinert, P. M. \& Steven, A. C. (1985) Nature (London) 316, 767

Steinert, P. M., Steven, A. C. \& Roop, D. R. (1985) Cell (Cambridge, Mass.) 42, 411-419

Weber, K. (1986) Nature (London) 320, 402

Zackroff, R. V., Goldman, A. E., Jones, J., Steinert, P. M. \& Goldman, R. D. (1984) J. Cell Biol. 98, 1231-1237 